\title{
The quest for the carbene bent-pentadiynylidene isomer of $\mathrm{C}_{5} \mathrm{H}_{2}$
}

\author{
Venkatesan S. Thimmakondu* \\ Department of Chemistry, Birla Institute of Technology and Science, Pilani, K K Birla Goa Campus, Goa - 403 \\ 726, India. \\ Amir Karton* \\ School of Molecular Sciences, The University of Western Australia, Perth, Western Australia 6009, Australia.
}

\begin{abstract}
The equilibrium geometry of the singlet ground electronic state of the bent isomer of $\mathrm{C}_{5} \mathrm{H}_{2}$, bentpentadiynylidene $\left(4 ; \widetilde{X}^{1} A_{1} ; C_{2 v}\right)$, has been theoretically investigated by means of the highly accurate W3-F12 thermochemical protocol. Five isomers of $\mathrm{C}_{5} \mathrm{H}_{2}$, namely linear-pentadiynylidene $\left(\mathbf{1} ; \widetilde{X}^{3} \Sigma_{g}^{-} ; D_{\infty h}\right)$, ethynylcyclopropenylidene $\left(2 ; \widetilde{X}^{1} A^{\prime} ; C_{s}\right)$, pentatetraenylidene $\left(\mathbf{3} ; \widetilde{X}^{1} A_{1} ; C_{2 v}\right)$, ethynylpropadienylidene $\left(\mathbf{5} ; \widetilde{X}^{1} A^{\prime} ; C_{s}\right)$, and 3-(didehydrovinylidene)cyclopropene $\left(\mathbf{6} ; \widetilde{X}^{1} A_{1} ; C_{2 v}\right)$ had already been identified in the laboratory. With respect to $\mathbf{1}$, the relative energy difference calculated at the CCSDT(Q)/CBS level of theory including zero-point vibrational energy corrections are: $0.66(\mathbf{2}), 13.53(\mathbf{3}), 14.12(\mathbf{4}), 15.40(5)$, and $20.01(6) \mathrm{kcal} \mathrm{mol}^{-1}$, respectively. Isomers 2-6 are associated with a non-zero dipole moment $(\mu \neq 0)$, however, except $\mathbf{4}$, all the other four isomers were identified by Fourier Transform Microwave spectroscopy, including $\mathbf{5}$ and $\mathbf{6}$ which lie higher in energy. Isomer 4 remains elusive to date. We believe that the theoretical data such as, optimal geometry, dipole moment, rotational and centrifugal distortion constants, harmonic vibrational frequencies, infra-red intensities, and isotopic shifts $\left({ }^{12} \mathrm{C}-{ }^{13} \mathrm{C}\right)$ in harmonic vibrational frequencies presented here would assist experimentalists in the identification this elusive molecule (4).

Keywords: $\operatorname{CCSDT}(\mathrm{Q}), \mathrm{W} 3$ theory, acetylenic carbenes, $\mathrm{C}_{5} \mathrm{H}_{2}$ isomers, Relative energies, microwave spectroscopy
\end{abstract}

Cite as:

V. S. Thimmakondu, A. Karton, Chem. Phys. 515, 411-417 (2018). https://doi.org/10.1016/j.chemphys.2018.04.023

\footnotetext{
${ }^{*}$ Corresponding authors

Email addresses: venkateshtsv@gmail.com (Venkatesan S. Thimmakondu), amir.karton@uwa.edu.au (Amir Karton)
}

Preprint submitted to Chemical Physics

April 23, 2018 


\section{Introduction}

Five isomers of $\mathrm{C}_{5} \mathrm{H}_{2}$ have been identified in the laboratory to date $[1,2,3,4,5,6,7]$. The open-shell triplet isomer, linear-pentadiynylidene (1), was initially claimed to be observed in a $5 \mathrm{~K}$

neon matrix by Maier and co-workers [1] on the basis of $\mathbf{1}^{3} \Sigma_{u}^{-} \leftarrow{ }^{{ }^{3}} \Sigma_{g X}^{-}$electronic transitions being recorded. However, due to lower singal-to-noise ratio for the electronic spectrum of $\mathbf{1}$, their spectral assignments were later challenged by McMahon and co-workers [5]. Isomer $\mathbf{1}$ of $\mathrm{C}_{5} \mathrm{H}_{2}$ was first unambigously identified by the photolysis of 1-diazo-2,4-pentadiyne in a $\mathrm{N}_{2}$ matrix and the electronic structure is characterized by various (IR, EPR, UV-vis) spectroscopic techniques by McMahon and co-workers [5]. Recently, the gas phase detection and resonant two-color two-photon ionization (R2C2PI) characterization of $\mathbf{1}$ was also reported by Maier and co-workers [6]. On the other hand, four closed-shell carbene isomers of $\mathrm{C}_{5} \mathrm{H}_{2}(\mathbf{2}, \mathbf{3}, \mathbf{5}$, and $\mathbf{6}$; Scheme 1), whose dipole mo-ments are nonzero $(\mu \neq 0)$, were identified by McCarthy and co-workers using Fourier Transform Microwave (FTMW) spectroscopy [2, 3, 4]. Among these molecules, the cumulene carbene isomer pentatetraenylidene (3), was reported initially [2]. Later, the laboratory detection of the threemembered ring-chain isomer, ethynylcyclopropenylidene (2) was also reported with both $a$ - and $b$ type rotational transitions as the inertial axis dipole moment components are in two directions for this molecule [3]. Both of these molecules are higher homologues of propadienylidene (a cumu-lene carbene of $\mathrm{C}_{3} \mathrm{H}_{2}$ ) and cyclopropenylidene, respectively. Also, the later are known to exist in the interstellar medium (ISM) $[8,9]$. The laboratory detection of these two molecules was helpful in the identification of the same in the ISM $[8,10]$. Recently, the di-deuterated derivative of cyclopropenylidene $\left(\mathrm{C}_{3} \mathrm{D}_{2}\right)$ was also identified in the ISM [11]. Computational studies using coupled-cluster the-ory by Stanton and co-workers [12] suggested that ethynylpropadienylidene (5), whose inertial axis dipole moment components are also in two directions, and 3-(didehydrovinylidene)cyclopropene (6) are also low-lying isomers (within $1 \mathrm{eV}$ ) on the $\mathrm{C}_{5} \mathrm{H}_{2}$ potential-energy surface (PES). The charge reversal and neutralization reionization mass spectra had not only detected $\mathbf{5}$ but also $\mathbf{1}$ and $\mathbf{3}$ via the corresponding anion [7]. Both of these theoretically suggested molecules (5 and $\mathbf{6})$, which are highly polar, were also detected later using the FTMW spectroscopy [4]. Our theoretical calcula-tions here suggest that there exists another closed-shell carbene isomer (4) on the low-energy side of the $\mathrm{C}_{5} \mathrm{H}_{2}$ PES, which may have been overlooked in the previous experimental $[1,2,3,4,5,6,7]$ and theoretical studies $[12,13,14,15,16,17,18,19,20,21,22]$.

From the electronic structure point of view, $\mathbf{4}$ can be considered as an acetylenic carbene, 
which are well-known intermediates in many prototypical organic reactions $[5,23,24,25,26,27$, $28,29,30,31]$. For the lower homologue, propynylidene (or propargylene diradical), there was a long-standing discrepancy between theory and experiment regarding the determination of the equilibrium geometry and its symmetry [32]. Eventually, this was settled to be a triplet groundstate $\left(\widetilde{X}^{3} B\right)$ with a $C_{2}$ symmetry $[32,33,34]$. Gottlieb and co-workers had stated that the detection of a quasi-linear triplet isomer of $\mathrm{C}_{5} \mathrm{H}_{2}$ would be intriguing as it would produce a nearly harmonic rotational spectrum with lines in the centimeter band very favorable for observation both in the laboratory and in the ISM [4]. This suggestion was initially ruled out as there is no minimum in the triplet PES of $\mathrm{C}_{5} \mathrm{H}_{2}$, which corresponds to a bent geometry. However, in the present work we focused on a bent geometry (4) on the singlet PES of $\mathrm{C}_{5} \mathrm{H}_{2}$. Although the dipole moment of 4 is smaller than that of 5 and 6 (Scheme 1), it still has an appreciable dipole moment of 2.08 Debye. Therefore, in principle, it is a potential candidate for detection by FTMW spectroscopy. The bent geometry (4) was initially found to be stable at the MP2/6-31G(d) level of theory and also at the QCISD/6-31G(d) level of theory $[12,13]$. However, it was thought to become linear with coupledcluster methods [12]. On the contrary, Mebel and co-workers had found it to be a local minimum at the B3LYP/6-311G(d,p) level of theory [21]. Here we revisit the electronic calculations at the relativistic, all-electron CCSDT(Q)/CBS level of theory by means of the W3-F12 thermochemical protocol [35] for isomers 1-6. The geometry optimizations and ZPVE corrections are calculated at the fc-CCSD(T)/cc-pVTZ level of theory.

Finally, we note that five $\mathrm{C}_{5} \mathrm{H}_{2}$ isomers have been detected in the laboratory to date $[1,2,3$, $4,5,6,7]$, although theoretically 38 isomers have been proposed in the past at the UB3LYP/6$311 \mathrm{G}(\mathrm{d}, \mathrm{p})$ level of theory [21, 22]. Nevertheless, isomer 4 was not studied in detail and also its spectroscopic properties are missing in the literature although it lies on the low-energy side of the PES. Also, none of the $\mathrm{C}_{5} \mathrm{H}_{2}$ isomers have been identified in space to date though structurally similar molecules have been detected in the past $[2,8,9,36]$. Here, we focus our attention on isomer 4 considering the amount of work done in the laboratory for the low-lying isomers of $\mathrm{C}_{5} \mathrm{H}_{2}$ $[1,2,3,4,5,6,7]$. We examine the bent acetylenic closed-shell carbene (4), which lies lower in energy than the two experimentally known isomers (5 and 6 ). We believe that our accurate theoretical data (such as the optimal geometry parameters, rotational constants, dipole moments, centrifugal distortion constants, harmonic vibrational frequencies, infrared intensities, and ${ }^{12} \mathrm{C}-{ }^{13} \mathrm{C}$ isotopic shifts) would assist the efforts of the experimentalists in the future and may compliment 


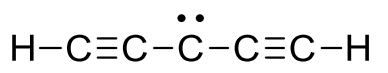

1

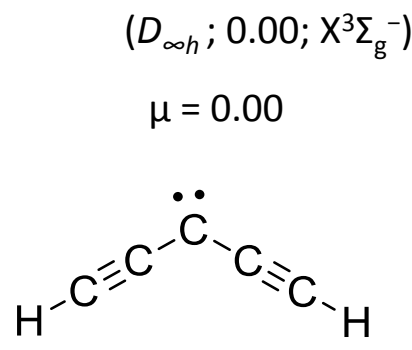

4

$$
\begin{aligned}
& \left(C_{2 v} ; 14.12 ; \mathrm{X}^{1} \mathrm{~A}_{1}\right) \\
& \mu=2.08
\end{aligned}
$$<smiles>C#CC1=C[CH]1</smiles>

2

$\left(C_{s} ; 0.66 ; \mathrm{X}^{1} \mathrm{~A}^{\prime}\right)$

$\mu=3.52$<smiles>C#CC=C=C</smiles>

5

$$
\begin{aligned}
& \left(C_{s} ; 15.40 ; X^{1} A^{\prime}\right) \\
& \mu=4.74
\end{aligned}
$$

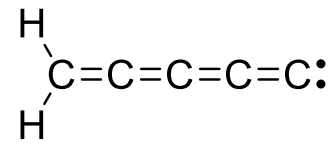

3

$\left(C_{2 v} ; 13.53 ; \mathrm{X}^{1} \mathrm{~A}_{1}\right)$

$\mu=5.81$<smiles>[CH]=C=C=C1C=C1</smiles>

6

$\left(C_{2 v} ; 20.01 ; \mathrm{X}^{1} \mathrm{~A}_{1}\right)$

$\mu=8.13$

Scheme 1: Isomers of $\mathrm{C}_{5} \mathrm{H}_{2}$ considered in the present work. Relative ZPVE-corrected energies are calculated at the $\operatorname{CCSDT}(\mathrm{Q}) / \mathrm{CBS}$ level of theory (in kcal $\mathrm{mol}^{-1}$ ). Dipole moments (in Debye) are computed at the CCSD(T)/ccpVTZ level of theory. Except 4, all other isomers are experimentally detected. Isomer $\mathbf{1}$ is a triplet and all others are singlets.

the experimental observations for this important intermediate (4) of $\mathrm{C}_{5} \mathrm{H}_{2}$.

\section{Computational Details}

All the isomers of $\mathrm{C}_{5} \mathrm{H}_{2}$ (1-6) reported in this study were optimized using coupled-cluster (CC) methods. The considered methods were CC with singles and doubles (CCSD) [37, 38], and CCSD with perturbative triple excitations $(\operatorname{CCSD}(\mathrm{T}))[39,40,41]$. Dunning's correlation consistent polarized valence double and triple zeta (cc-pVnZ; $n=\mathrm{D}$ and $\mathrm{T}$ ) basis sets [42] were used in these calculations. The carbon $1 s$ orbitals were frozen in the post-Hartree-Fock (HF) calculations. Harmonic vibrational frequencies were calculated at the same levels of theory by analytic calculation 
of second derivatives [43] for all stationary points. For isomer $\mathbf{1}$ both unrestricted HF (UHF) and restricted open-shell HF (ROHF) wavefunctions were used at the fc-CCSD(T)/cc-pVTZ level of theory since the ground electronic state is a triplet $\left(\widetilde{X}^{3} \Sigma_{g}^{-}\right)$. In addition to the CC calculations, for isomer 4 we have also done our optimizations and harmonic vibrational frequencies at the second-order Møller-Plesset perturbation theory (MP2) [44] to see how comparable the geometries are with respect to $\mathrm{CC}$ methods. For isomer 4, the anion geometry $\left(\mathrm{C}_{5} \mathrm{H}_{2}^{-}\right)$is optimized at the UCCSD(T)/aug-cc-pVDZ level of theory and one-dimensional potential energy scan along the $\mathrm{C}_{1} \mathrm{C}_{2} \mathrm{C}_{3}$ bending coordinate for the anion and neutral (singlet and triplet electronic states) were calculated to determine the barrier to linearity. We have also done HF stability analysis $[45,46,47]$ for all optimized geometries obtained at the fc-CCSD $(\mathrm{T}) / \mathrm{cc}-\mathrm{pVTZ}$ level of theory to find out instability of the wavefunction and/or if there are small eigenvalues in the orbital rotation Hessian. We also calculated the so-called \% TAE $[(\mathrm{T})]$ diagnostics for all isomers $[48,49]$ to estimate the significance of contributions from post-CCSD(T) excitations. All the above electronic structure calculations were carried out with the CFOUR program package [50].

In order to obtain accurate relative energies, high-level benchmark data have been obtained using W3-F12 theory [35]. W3-F12 theory (and its earlier version W3.2 theory [48]) represent layered extrapolations to the all-electron CCSDT(Q)/CBS limit and can achieve near-benchmark accuracy for atomization reactions (i.e., they are associated with root-mean-square deviations, RMSDs, from accurate atomization energies smaller than $1 \mathrm{~kJ} \mathrm{~mol}^{-1}$ ). W3-F12 theory combines F12 methods with basis-set extrapolations in order to reproduce the CCSDT(Q)/CBS energy. In W3-F12, the $\operatorname{CCSD}(\mathrm{T}) / \mathrm{CBS}$ energy is obtained from W2-F12 theory, [35] and the post-CCSD(T) contributions are obtained from W3.2 theory [48]. The computational protocols of the Wn-F12 theories have been specified and rationalized in great detail in ref. [35] (see ref. [49] for a recent review). In brief, the HF component is calculated with the cc-pVQZ-F12 basis set of Peterson et al., [51, 52] which was developed for explicitly correlated calculations. The valence CCSD-F12 correlation energy is extrapolated from the cc-VTZ-F12 and cc-VQZ-F12 basis sets. The quasiperturbative triples, (T), corrections are calculated with the cc-pVTZ-F12 basis set. The higher-order connected triples $(\operatorname{CCSDT}-\mathrm{CCSD}(\mathrm{T}))$ valence correlation contribution is extrapolated from the cc-pVDZ and ccpVTZ basis sets. The parenthetical connected quadruples contribution (CCSDT(Q)-CCSDT) is calculated with the cc-pVDZ basis set. The CCSD inner-shell contribution is calculated with the core-valence weighted correlation-consistent aug-cc-pwCVTZ basis set of Peterson and Dunning, 
[53] whilst the (T) inner-shell contribution is calculated with the cc-pwCVTZ basis set without the $f$ functions. Finally, the scalar relativistic contribution (in the second-order Douglas-Kroll-Hess approximation $[54,55])$ is obtained as the difference between non-relativistic $\operatorname{CCSD}(\mathrm{T}) /$ aug'-cc-pVDZ and relativistic CCSD(T)/aug'-cc-pVDZ-DK calculations (where, aug' indicates the combination of a non-augmented basis set on $\mathrm{H}$ and an augmented basis set on $\mathrm{C}$ ) [56]. The calculations in W3-F12 were carried out with the MOLPRO (all CCSD $(\mathrm{T})$ calculations) and the MRCC (all post-CCSD $(\mathrm{T})$ calculations) program suites. $[57,58]$

\section{Results and Discussion}

The component breakdown of the W3-F12 energies as well as the final relativistic, all-electron, $\operatorname{CCSDT}(\mathrm{Q}) / \mathrm{CBS}$ relative energies on the electronic surface $\left(\Delta E_{e}\right)$ and at $0 \mathrm{~K}\left(\Delta E_{0}\right)$ are given in Table 1. It also depicts relative energies obtained from previous theoretical works $[6,12,21]$. Let us begin by considering the components of W3-F12 theory on the electronic PES $\left(\Delta E_{e}\right)$. Inspection of the HF/CBS and CCSD/CBS components of the relative energies reveals that, for all isomers apart from 2, the CCSD correlation component is significantly larger than the SCF component. The $(\mathrm{T})$ correlation component has significant contributions ranging (in absolute value) from 0.11 (6) to $2.74(\mathbf{3}) \mathrm{kcal} \mathrm{mol}^{-1}$. The higher-order connected triple excitations systematically reduce the relative energies by chemically significant amounts ranging from $0.06(2)$ to $0.59(4) \mathrm{kcal} \mathrm{mol}^{-1}$. The (Q) correlation component still has significant contributions ranging (in absolute value) from 0.11 (5) to $0.48(\mathbf{2}) \mathrm{kcal} \mathrm{mol}^{-1}$. Overall, the post-CCSD(T) contributions affect the relative energies by moderate amounts ranging (in absolute value) from $0.20(\mathbf{5})$ to $0.81(\mathbf{4}) \mathrm{kcal} \mathrm{mol}^{-1}$. We note in passing that these contributions are consistent with the $\% \mathrm{TAE}(\mathrm{T})$ diagnostics, which range between $2.6(\mathbf{2})$ to $3.2(\mathbf{3})$ (Table 2). Moreover, the largest \% TAE(T) values are obtained for the two isomers with the largest overall post-CCSD(T) contributions, namely isomers 3 and $\mathbf{4}$ with post-CCSD(T)

contributions of -0.71 and $-0.81 \mathrm{kcal} \mathrm{mol}^{-1}$, respectively. Finally, we note that the core-valence component reduces the relative energies by chemically significant amounts ranging from 0.51 (4) to $0.86(5) \mathrm{kcal} \mathrm{mol}^{-1}$ and the scalar relativistic contributions still reduce the relative energies by about $0.1 \mathrm{kcal} \mathrm{mol}^{-1}$ in all cases.

Let us consider the results on the electronic PES $\left(\Delta E_{e}\right)$. At the relativistic, all-electron $\operatorname{CCSD}(\mathrm{T}) / \mathrm{CBS}$ and CCSDT(Q)/CBS levels of theory isomer $\mathbf{2}$ is the most stable isomer. In particular, it is more stable than isomer $\mathbf{1}$ by as much as 2.73 and $2.31 \mathrm{kcal} \mathrm{mol}^{-1}$, respectively. 
Upon inclusion of the ZPVE corrections this order is reversed and isomer $\mathbf{1}$ becomes more stable than isomer 2 by 0.24 and $0.66 \mathrm{kcal} \mathrm{mol}^{-1}$ at the relativistic, all-electron $\operatorname{CCSD}(\mathrm{T}) / \mathrm{CBS}$ and CCSDT(Q)/CBS levels of theory, respectively.

Our best relativistic, all-electron CCSDT(Q)/CBS relative $\Delta E_{0}$ values are: $0.66(\mathbf{2}), 13.53(\mathbf{3})$, $14.12(4), 15.40(5)$, and $20.01(6) \mathrm{kcal} \mathrm{mol}^{-1}$. These values predict the same isomer ordering as the all-electron $\operatorname{CCSD}(\mathrm{T}) / \mathrm{cc}-\mathrm{pVTZ}$ values of Stanton and co-workers [12]. However, our relative energies are systematically larger (Table 1). In particular, our best CCSDT(Q)/CBS values are larger by $1.35(\mathbf{2}), 0.29(\mathbf{3}), 1.38(5)$, and $1.13(\mathbf{6}) \mathrm{kcal} \mathrm{mol}^{-1}$ compared to the previous CCSD(T)/ccpVTZ values [12]. Considering the magnitude of the relative energies, the difference between the two sets of values is particularly pronounced for isomer $\mathbf{2}$, which we predict is much closer in energy to isomer 1 (i.e., 0.66 vs. $2.01 \mathrm{kcal} \mathrm{mol}^{-1}$, Table 1). The differences in relative energy (Table 1) between our values and the values of Stanton and co-workers [12] could be attributed to three main reasons (i) we have estimated our relative energies using a ROHF based wavefunction for isomer $\mathbf{1}$, whereas they have estimated their relative energies using a UHF based wavefunction for isomer 1; (ii) our best values are calculated at the relativistic, all-electron CCSDT(Q)/CBS levels of theory, whereas their values are calculated at the all-electron $\operatorname{CCSD}(\mathrm{T}) / \mathrm{cc}-\mathrm{pVTZ}$ level of theory; and (iii) we have calculated our ZPVE corrections at the fc-CCSD(T)/cc-pVTZ level of theory, whereas they have done their ZPVE corrections at a lower level of theory (ae-CCSD/DZP).

Comparison of our best relativistic, all-electron $\operatorname{CCSDT}(\mathrm{Q}) / \operatorname{CBS}$ relative $\Delta E_{0}$ energies with the M06-2X/cc-pVTZ values of Maier and co-workers [6] reveals that M06-2X does not predict the correct isomer order. In particular, M06-2X predicts that isomer $\mathbf{2}$ is the most stable isomer and that isomer 1 lies higher in energy by $0.60 \mathrm{kcal} \mathrm{mol}^{-1}$. In addition, it predicts that isomer $\mathbf{6}$ is more stable than isomer $\mathbf{5}$ and essentially isoenergetic with isomer $\mathbf{3}$. This indicates that M06-2X is not a reliable method for calculating these relative energies. Similarly, relative energies obtained at the CCSD(T)/cc-pVQZ//B3LYP/6-311G(d,p) level of theory predicts that isomer $\mathbf{2}$ is more stable than 1 by $1.50 \mathrm{kcal} \mathrm{mol}^{-1}$ [21]. However, we affirm that at the CCSDT(Q)/CBS level of theory including zero-point vibrational energy (ZPVE) corrections at the fc-CCSD(T)/cc-pVTZ level of theory, the linear isomer (1) is the most stable structure (Table 1) on the $\mathrm{C}_{5} \mathrm{H}_{2}$ PES. The poor performance of density functional theory (DFT) for similar relative energies has been previously noted in the case of $\mathrm{C}_{7} \mathrm{H}_{2}$ isomers, in particular for the low-lying non-linear isomers [59, 60, 61, 62] (see also ref. [63] for a comprehensive survey of the performance of a wide range of DFT methods 
for challenging structural relative energies). We note that we have tried to optimize the geometry of isomer 1 in the singlet electronic state $\left(\widetilde{X}^{1} A_{g}\right)$ at the fc-CCSD(T)/cc-pVTZ level of theory [64], however, this optimization resulted in multiple imaginary frequencies (see Supporting Information for further details).

Now let us turn to the structural and spectroscopic properties of the $\mathrm{C}_{5} \mathrm{H}_{2}$ isomers. The atom numbering scheme we have adopted for all isomers (1-6) of $\mathrm{C}_{5} \mathrm{H}_{2}$ is shown in Figure 1. The optimal geometry parameters, dipole moment, rotational and centrifugal distortion constants obtained at six different levels for isomer 4 are shown in Table 3. Since the electronic structure of other isomers $(\mathbf{1 - 3}, \mathbf{5}$, and $\mathbf{6})$ are discussed in detail in other works [6,12], we will not discuss them here apart from making comparisons with relative energies from the previous investigations. Harmonic vibrational frequencies estimated at the fc-CCSD $(\mathrm{T}) / \mathrm{cc}-\mathrm{pVTZ}$ level of theory for isomer $\mathbf{4}$ is given in Table 4. Cartesian coordinates of the optimized geometries at all levels of theory for all the isomers investigated are given as Supporting Information.

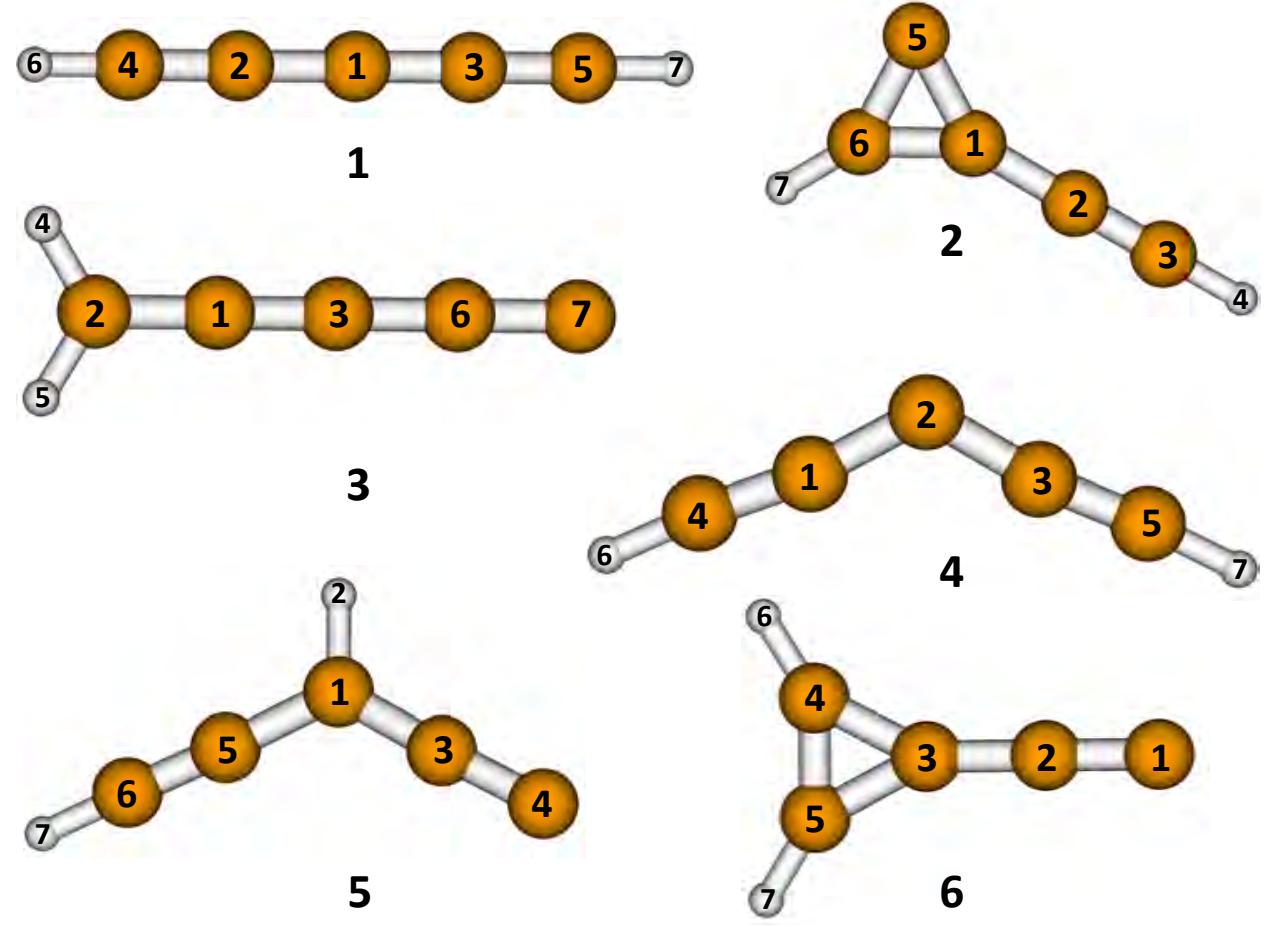

Figure 1: Atom numbering scheme we have adopted for isomers $\mathbf{1}$ to $\mathbf{6}$ of $\mathrm{C}_{5} \mathrm{H}_{2}$.

The optimal geometry of 4 collected in Table 3 at all levels tells us that the bond lengths of $\mathrm{C}_{1} \mathrm{C}_{4}$ 
and $\mathrm{C}_{3} \mathrm{C}_{5}$ are shorter with triple bond characteristics. On the contrary, the bond lengths of $\mathrm{C}_{1} \mathrm{C}_{2}$ and $\mathrm{C}_{2} \mathrm{C}_{3}$ are not perfectly reminiscent of single bond characteristics. The presence of a lone-pair of electrons on $\mathrm{C}_{2}$ is evidently seen in the bond distances of the later as it is somewhat shorter compared to typical C-C single bonds. Also, the usual trend of contraction of bond lengths when the basis set is increased within a particular method is seen for this molecule as well. Moreover, it is worth mentioning here that the bond lengths are systematically overestimated at the MP2, CCSD, and CCSD $(\mathrm{T})$ levels with respect to cc-pVDZ basis set. These are largely due to lack of higher angular momentum polarization functions $[65,66,67,68,69,70]$ and are consistent with the previous observations [62]. As far as bond angles are concerned, all of them are expanded when the basis set size is increased within a particular method. The nearly 120 degree bond angle obtained at all levels for $\mathrm{C}_{1} \mathrm{C}_{2} \mathrm{C}_{3}$ confirms that the molecule is bent.

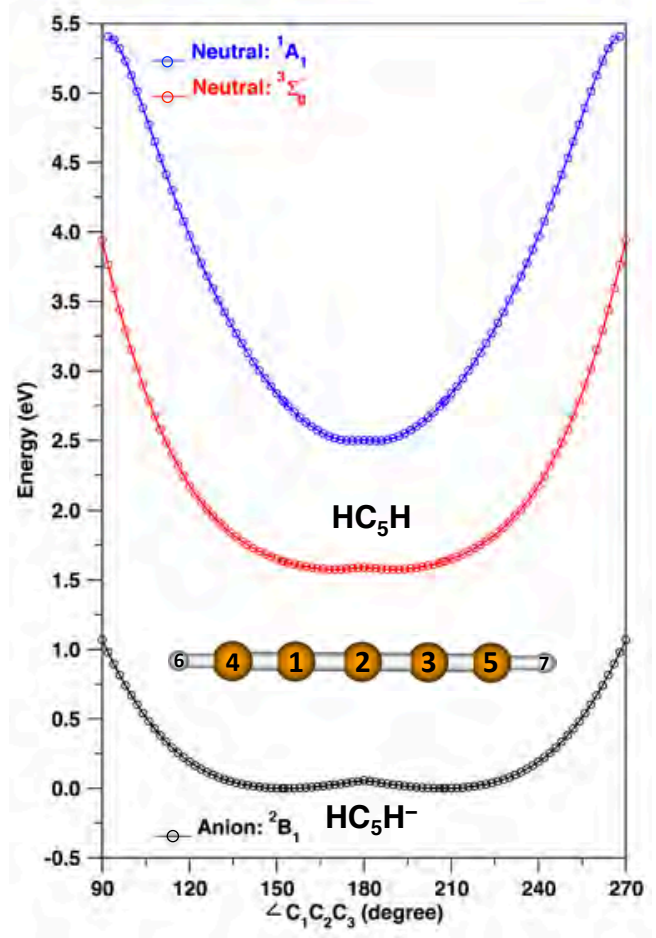

Figure 2: One-dimensional potential-energy scan along the $\mathrm{C}_{1} \mathrm{C}_{2} \mathrm{C}_{3}$ bending coordinate of isomer 4 calculated at the $\operatorname{UCCSD}(\mathrm{T})$ /aug-cc-pVDZ level of theory. Energies are given relative to the minimum of the bent-pentadiynylidene anion surface. All bond angles except $\angle \mathrm{C}_{1} \mathrm{C}_{2} \mathrm{C}_{3}$ are approximated to be linear, while the other values are set to the values that minimize the energy of the anion under these constraints.

The rotational constants $\left(A_{e}, B_{e}\right.$, and $\left.C_{e}\right)$ obtained at all levels indicate that isomer 4 is an 
asymmetric top. At the fc-CCSD(T)/cc-pVTZ level of theory the dipole moment is 2.08 Debye. As discussed above, this structure lies $14.12 \mathrm{kcal} \mathrm{mol}^{-1}$ above 1 but 1.28 and $5.89 \mathrm{kcal} \mathrm{mol}^{-1}$ below the energies of the already known isomers 5 and $\mathbf{6}$. Nevertheless, 4 remains as an elusive molecule to date. We note that the dipole moment of $\mathbf{4}$ is smaller than that of the other isomers, which are already detected with FTMW spectroscopy. Nevertheless, a dipole moment of 2.08 Debye makes it a potential candidate for detection by FTMW spectroscopy. In addition, for $\mathbf{2}$ and $\mathbf{5}$, the inertial axis dipole moment components are in two directions whereas for $\mathbf{3}, \mathbf{4}$, and $\mathbf{6}$, it is in only one direction. This means that only one type of rotational transition is possible for the later whereas for the former, both $a$ - and $b$-type rotational transitions are possible. The highly intense vibrational mode for 4 turns out to be the asymmetric $\mathrm{C} \equiv \mathrm{C}$ stretching of $b_{2}$ symmetry whose harmonic frequency is $1974 \mathrm{~cm}^{-1}$ at the $\mathrm{fc}-\mathrm{CCSD}(\mathrm{T}) / \mathrm{cc}-\mathrm{pVTZ}$ level of theory (Table 4 ). There are three different carbon atoms for 4 whose isotopic shifts $\left({ }^{12} \mathrm{C}-{ }^{13} \mathrm{C}\right)$ in harmonic vibrational frequencies are also given in Table 4 . We believe that this data would be helpful in identifying this hypothetical molecule in the laboratory.

In response to a comment made by one of the reviewers of the present manuscript regarding the photoelectron spectra of $\mathrm{HC}_{4} \mathrm{~N}^{-}$[71], we calculated the one-dimentional potential energy scan along one of the bending coordinates $\left(\mathrm{C}_{1} \mathrm{C}_{2} \mathrm{C}_{3}\right)$ of 4 at the UCCSD(T)/aug-cc-pVDZ level of theory. The anion of 4 , which is isoelectronic to $\mathrm{HC}_{4} \mathrm{~N}^{-}$, was optimized at the later level and used as a reference for the bending potentials of the neutral triplet $\left({ }^{3} \Sigma_{g}^{-}\right)$and singlet $\left({ }^{1} A_{1}\right)$ electronic states. These results are shown in Figure 2. Unlike in $\mathrm{HC}_{4} \mathrm{~N}$, where singlet-triplet crossing was observed upon bending, we do not notice the same in $\mathrm{C}_{5} \mathrm{H}_{2}$ upon photodetachment. Also, the barrier to linearity from the one-dimensional scan on the neutral triplet electronic state was found to be $16.5 \mathrm{meV}$ at the UCCSD(T)/aug-cc-pVDZ level of theory. Like in $\mathrm{HC}_{4} \mathrm{~N}$, where the barrier to linearity was found to be $24 \mathrm{meV}$ at the ROMP2/6-311++G(d,p) level of theory [71], the potential of the triplet electronic state in $\mathrm{C}_{5} \mathrm{H}_{2}$ was found to be extremely floppy. This scenario suggests that isomerization of $\mathbf{4}$ to $\mathbf{1}$ may proceed very fast.

Nevertheless, among the low-lying closed-shell carbenes identified thus far, $\mathbf{4}$ is certainly less polar. Although thermodynamically more stable than $\mathbf{5}$ and $\mathbf{6}$, it remains elusive to date. Therefore, the kinetic stability of $\mathbf{4}$ is an important question here. Also, considering the proximity in energy of isomers 3-5 (i.e., within $1.91 \mathrm{kcal} \mathrm{mol}^{-1}$ at the CCSDT(Q)/CBS level of theory, Table 1), we propose the following possibilities, apart from the relaxation of 4 to 1 : (i) dimerization of $\mathbf{4}$, (ii) 
$[1,3] \mathrm{H}$-transfer of $\mathbf{4}$ to $\mathbf{5}$, and (iii) [1,5] H-transfer of $\mathbf{4}$ to $\mathbf{3}$. These speculations require further investigations. Nevertheless, it is worth pointing here that point (i) may be likely considering the fact that the derivatives of acetylenic carbenes $\left(\mathrm{RC}_{5} \mathrm{R}\right)$ undergoing dimerization were reported in the literature in the past $[23,24,25]$.

\section{Conclusions}

The energetic, structural, and spectroscopic properties of a low-lying closed-shell carbene of $\mathrm{C}_{5} \mathrm{H}_{2}$ (4) have been investigated at high levels of theory. While a quasi-linear triplet geometry with $C_{2}$ symmetry was feasible for $\mathrm{C}_{3} \mathrm{H}_{2}$, in the case of $\mathrm{C}_{5} \mathrm{H}_{2}$, we conclude that both linear triplet (1) and bent-singlet (4) geometries are possible. At the relativistic, all-electron CCSDT(Q)/CBS level of theory including ZPVE corrections, these two isomers are energetically separated by $14.12 \mathrm{kcal}$ $\mathrm{mol}^{-1}$. It is worth noting here that five isomers (1-3, 5, and $\mathbf{6}$ ) of $\mathrm{C}_{5} \mathrm{H}_{2}$ are known to date experimentally. Isomer $\mathbf{4}$, which is energetically sandwiched between $\mathbf{3}$ and $\mathbf{5}$, remains elusive to date. In the current investigation we use the highly accurate W3-F12 thermochemical protocol to obtain the relative energy differences of these molecules. We conclude that $\mathbf{1}$ is the most stable isomer at the relativistic, all-electron CCSDT(Q)/CBS level of theory (including ZPVE corrections) and 2 lies only $0.66 \mathrm{kcal} \mathrm{mol}^{-1}$ above $\mathbf{1}$. These high-level results are in contrast with the DFT investigations $[6,21]$, which predicted that 2 is the most stable isomer of $\mathrm{C}_{5} \mathrm{H}_{2}$. The optimal geometry parameters, dipole moments, rotational constants, centrifugal distortion constants, harmonic vibrational frequencies and isotopic shifts in harmonic vibrational frequencies calculated at various levels of theory may assist efforts in the identification of this elusive intermediate in the laboratory if not in astronomical sources. Considering the fact that 4 is 1.28 and $5.89 \mathrm{kcal} \mathrm{mol}^{-1}$ more stable than the already known isomers $\mathbf{5}$ and $\mathbf{6}$, we believe that it might be possible to detect this molecule using FTMW spectroscopy. It is also worth noting here that detecting such elusive molecules are crucial in the identification of the same in astronomical sources, although its kinetic stability is still an open question. To this end, further investigations along the directions of (i) isomerization of 4 to $\mathbf{1}$; (ii) dimerization of $\mathbf{4}$; (iii) [1,3] H-transfer of $\mathbf{4}$ to $\mathbf{5}$, and (iv) [1,5] H-transfer of $\mathbf{4}$ to $\mathbf{3}$ are currently underway.

\section{Acknowledgments}

This article is written in honor of Prof. Wolfgang Domcke (Technical University of Munich) on 
the occasion of his 70th birthday. We gratefully acknowledge the generous allocation of computing time from the National Computational Infrastructure (NCI) National Facility. VST thanks Prof. John F. Stanton (University of Florida) for having helpful discussions during the early stages of this work and also Prof. T. P. Radhakrishnan (University of Hyderabad) for his interest in this work. VST also thanks Dr Mayavan Viji (Chungbuk National University, South Korea) for drawing the schemes with chemdraw and having a discussion in detail about carbene dimerization. A.K. acknowledges the Australian Research Council for a Future Fellowship (FT170100373).

[1] J. Fulara, P. Freivogel, D. Forney, J. P. Maier, J. Chem. Phys. 103 (1995) 8805-8810.

[2] M. C. McCarthy, M. J. Travers, A. Kovács, W. Chen, S. E. Novick, C. A. Gottlieb, P. Thaddeus, Science 275 (1997) 518-520.

[3] M. J. Travers, M. C. McCarthy, C. A. Gottlieb, P. Thaddeus, Astrophys. J. 483 (1997) L135L138.

[4] C. A. Gottlieb, M. C. McCarthy, V. D. Gordon, J. M. Chakan, A. J. Apponi, P. Thaddeus, Astrophys. J. 509 (1998) L141-L144.

[5] N. P. Bowling, R. J. Halter, J. A. Hodges, R. A. Seburg, P. S. Thomas, C. S. Simmons, J. F. Stanton, R. J. McMahon, J. Am. Chem. Soc. 128 (2006) 3291-3302.

[6] M. Steglich, J. Fulara, S. Maity, A. Nagy, J. P. Maier, J. Chem. Phys. 142 (2015) 244311-6.

[7] S. J. Blanksby, S. Dua, J. H. Bowie, D. Schröder, H. Schwarz, J. Phys. Chem. A 102 (1998) 9949-9956.

[8] P. Thaddeus, J. M. Vrtilek, C. A. Gottlieb, Astrophys. J. 299 (1985) L63-L66.

[9] J. Cernicharo, C. A. Gottlieb, M. Guelin, T. C. Killian, G. Paubert, P. Thaddeus, J. M. Vrtilek, Astrophy. J. Lett. 398 (1991) L39-L41.

[10] J. M. Vrtilek, C. A. Gottlieb, E. W. Gottlieb, T. C. Killian, P. Thaddeus, Astrophys. J. Lett. 364 (1990) L53-L56.

[11] S. Spezzano, S. Brünken, P. Schilke, P. Caselli, K. M. Menten, M. C. McCarthy, L. Bizzocchi, S. P. Trevinõ-Morales, Y. Aikawa, S. Schlemmer, Astrophys. J. Lett. 769 (2013) L19. 
[12] R. A. Seburg, R. J. McMahon, J. F. Stanton, J. Gauss, J. Am. Chem. Soc. 119 (1997) 1083810845.

[13] R. A. Seburg, Ph.D. Dissertation. University of Wisconsin-Madison, Madison, WI, 1995.

[14] D. L. Cooper, S. C. Murphy, Astrophy. J. 333 (1988) 482-490.

[15] Q. Fan, G. V. Pfeiffer, Chem. Phys. Lett. 162 (1989) 472-478.

[16] A. Mavrandonakis, M. Mühlhäuser, G. E. Froudakis, S. D. Peyerimhoff, Phys. Chem. Chem. Phys. 4 (2002) 3318-3321.

[17] L. Horný, N. D. K. Petraco, H. F. Schaefer III, J. Am. Chem. Soc. 124 (2002) 14716-14720.

[18] I. Alkorta, J. Elguero, Struc. Chem. 16 (2005) 77-79.

[19] L. Veis, P. Čársky, J. Pittner, J. Michl, Collect. Czech. Chem. Commun. 73 (2008) 1525-1551.

[20] Y. Zhang, L. Wang, Y. Li, J. Zhang J. Chem. Phys. 138 (2013) 204303-9.

[21] A. M. Mebel, G. -S. Kim, V. V. Kislov, R. I. Kaiser, J. Phys. Chem. A 111 (2007) 6704-6712.

[22] B. J. Sun, C. Y. Huang, H. H. Kuo, K. T. Chen, H. L. Sun, C. H. Huang, M. F. Tsai, C. H. Kao, Y. S. Wang, L. G. Gao, R. I. Kaiser, A. H. H. Chang, J. Chem. Phys. 128 (2008) 244303.

[23] Y. Hori, K. Noda, S. Kobayashi, H. Taniguchi, Tetrahedron Lett. 40 (1969) 3563.

[24] H. Hauptmann, Tetrahedron 32 (1976) 1293-1297.

[25] M. Noro, N. Koga, H. Iwamura, J. Am. Chem. Soc. 115 (1993) 4916.

[26] J. T. DePinto, R. J. McMahon, J. Am. Chem. Soc. 115 (1993) 12573-12574.

[27] J. T. DePinto, W. A. deProphetis, J. L. Menke, R. J. McMahon, J. Am. Chem. Soc. 129 (2007) $2308-2315$

[28] P. S. Thomas, N. P. Bowling, R. J. McMahon, J. Am. Chem. Soc. 131 (2009) 8649-8659.

[29] P. S. Thomas, N. P. Bowling, N. J. Burrmann, R. J. McMahon, J. Org. Chem. 75 (2010) $6372-6381$. 
[30] N. P. Bowling, N. J. Burrmann, R. J. Halter, J. A. Hodges, R. J. McMahon, J. Org. Chem. 75 (2010) 6382-6390.

[31] S. N. Knezz, T. A. Waltz, B. C. Haenni, N. J. Burrmann, R. J. McMahon, J. Am. Chem. Soc. 138 (2016) 12596-12604.

[32] R. A. Seburg, E. V. Patterson, J. F. Stanton, R. J. McMahon, J. Am. Chem. Soc. 119 (1997) $5847-5856$.

[33] D. L. Osborn, K. M. Vogelhuber, S. W. Wren, E. M. Miller, Y. -J. Lu, A. S. Case, L. Sheps, R. J. McMahon, J. F. Stanton, L. B. Harding, B. Ruscic, W. C. Lineberger, J. Am. Chem. Soc. 136 (2014) 10361-10372.

[34] R. A. Seburg, E. V. Patterson, R. J. McMahon, J. Am. Chem. Soc. 131 (2009) 9442-9455.

[35] A. Karton, J. M. L. Martin, J. Chem. Phys. 136 (2012) 124114.

[36] W. D. Langer, T. Velusamy, T. B. H. Kuiper, R. Peng, M. C. McCarthy, M. J. Travers, A. Kovács, C. A. Gottlieb, P. Thaddeus, Astrophy. J. 480 (1997) L63-L66.

[37] G. D. Purvis III, R. J. Bartlett, J. Chem. Phys. 76 (1982) 1910-1918.

[38] J. F. Stanton, J. Gauss, J. D. Watts, R. J. Bartlett, J. Chem. Phys. 94 (1991) 4334-4345.

[39] K. Raghavachari, G. W. Trucks, J. A. Pople, M. Head-Gordon, Chem. Phys. Lett. 157 (1989) 479-483.

[40] R. J. Bartlett, J. D. Watts, S. A. Kucharski, J. Noga, Chem. Phys. Lett. 165 (1990) 513-522.

[41] J. F. Stanton, Chem. Phys. Lett. 281 (1997) 130-134.

[42] T. H. Dunning, Jr., J. Chem. Phys. 90 (1989) 1007-1023.

[43] J. Gauss, J. F. Stanton, Chem. Phys. Lett. 276 (1997) 70-77.

[44] C. Møller, M. S. Plesset, Phys. Rev. 46 (1934) 618-622.

[45] J. Paldus, J. Č́̌žzek, J. Chem. Phys. 52 (1970) 2919.

[46] R. Seeger, J. A. Pople, J. Chem. Phys. 66 (1977) 3045. 
[47] T. D. Crawford, J. F. Stanton, W. D. Allen, H. F. Schaefer III, J. Chem. Phys. 107 (1997) 10626-10632.

[48] A. Karton, E. Rabinovich, J. M. L. Martin, B. Ruscic, J. Chem. Phys. 125 (2006) 144108-17.

[49] A. Karton, WIREs Comput. Mol. Sci. 6 (2016) 292-310.

[50] J. F. Stanton, J. Gauss, M. E. Harding, P. G. Szalay with contributions from A. A. Auer, R. J. Bartlett, U. Benedikt, C. Berger, D. E. Bernholdt, Y. J. Bomble, L. Cheng, O. Christiansen, M. Heckert, O. Heun, C. Huber, T. -C. Jagau, D. Jonsson, J. Jusélius, K. Klein, W. J. Lauderdale, D. A. Matthews, T. Metzroth, D. P. O’Neill, D. R. Price, E. Prochnow, K. Ruud, F. Schiffmann, W. Schwalbach, S. Stopkowicz, A. Tajti, J. Vázquez, F. Wang, J. D. Watts and the integral packages MOLECULE (J. Almlöf and P. R. Taylor), PROPS (P. R. Taylor), ABACUS (T. Helgaker, H. J. Aa. Jensen, P. Jørgensen, and J. Olsen), and ECP routines by A. V. Mitin and C. van Wüllen. For the current version, see http://www.cfour.de.

[51] K. A. Peterson, T. B. Adler, H.-J. Werner, J. Chem. Phys. 128 (2008) 084102.

[52] K. E. Yousaf, K. A. Peterson, J. Chem. Phys. 129 (2008) 184108.

[53] K. A. Peterson, T. H. Dunning, J. Chem. Phys. 117 (2002) 10548.

[54] M. Douglas, N. M. Kroll, Ann. Phys. 82 (1974) 89.

[55] B. A. Hess, Phys. Rev. A 33 (1986) 3742.

[56] W. A. de Jong, R. J. Harrison, D. A. Dixon, J. Chem. Phys. 114 (2001) 48.

[57] H.-J. Werner, P. J. Knowles, G. Knizia, F. R. Manby, M. Schütz, WIREs Comput. Mol. Sci. 2 (2012) 242 .

[58] Z. Rolik, L. Szegedy, I. Ladjánszki, B. Ladóczki, and M. Kállay, J. Chem. Phys. 139 (2013) 094105.

[59] S. Dua, S. J. Blanksby, J. H. Bowie, J. Phys. Chem. A, 104 (2000) 77-85.

[60] B. J. Sun, C. H. Huang, M. F. Tsai, H. L. Sun, L. G. Gao, Y. S. Wang, Y. Y. Yeh, Y. H. Shih, Z. F. Sia, P. H. Chen, R. I. Kaiser, A. H. H. Chang, J. Chem. Phys. 131 (2009) 104305-13. 
[61] V. S. Thimmakondu, Comput. Theoret. Chem. 1079 (2016) 1-10.

[62] V. S. Thimmakondu, A. Karton, Phys. Chem. Chem. Phys. 19 (2017) 17685-17697.

[63] A. Karton, J. M. L. Martin, Mol. Phys. 110 (2012) 2477.

[64] The singlet $\left(\widetilde{X}^{1} A_{g}\right)$ electronic state of isomer 1 was found to be a sixth-order saddle point at the fc-CCSD(T)/cc-pVTZ level of theory and lies at $19.61 \mathrm{kcal} \mathrm{mol}^{-1}$ (ZPVE corrected and scaled) above the triplet $\left(\widetilde{X}^{3} \Sigma_{g}^{-}\right)$ground electronic state of $\mathbf{1}$.

[65] P. R. Spackman, D. Jayatilaka, A. Karton, J. Chem. Phys. 145 (2016) 104101-10.

[66] D. Feller, K. A. Peterson, J. Chem. Phys. 126 (2007) 114105.

[67] S. Wang and H. F. Schaefer III, J. Chem. Phys. 124 (2006) 044303-8.

[68] K. L. Bak, J. Gauss, P. Jørgensen, J. Olsen, T. Helgaker, J. F. Stanton, J. Chem. Phys. 114 (2001) 6548 .

[69] J. M. L. Martin, P. R. Taylor, J. Phys. Chem. 100 (1996) 6047-6056.

[70] Y. Xie, G. E. Scuseria, B. F. Yates, Y. Yamaguchi, H. F. Schaefer, J. Am. Chem. Soc. 111 (1989) 5181-5185.

[71] K. M. Vogelhuber, S. W. Wren, C. J. Shaffer, R. J. McMahon, A. B. McCoy, W. C. Lineberger, J. Chem. Phys. 135 (2011) 204307-9.

[72] M. K. Kesharwani, B. Brauer, J. M. L. Martin, J. Phys. Chem. A, 119 (2015) 1701-1714. 
Table 1: Component breakdown of the W2-F12 and W3-F12 relative energies along with the final W2-F12 and W3-F12 values at the bottom of the well $\left(\Delta E_{e}\right)$ and at 0 $\mathrm{K}\left(\Delta E_{0}\right)$. All values are given relative to isomer 1 in $\mathrm{kcal} \mathrm{mol}^{-1}$.

\begin{tabular}{|c|c|c|c|c|c|c|}
\hline Theory & Comp. & 2 & 3 & 4 & 5 & 6 \\
\hline W2-F12 & $\mathrm{HF}$ & -18.77 & 4.88 & 2.66 & 1.89 & 1.98 \\
\hline W2-F12 & CCSD & 13.77 & 9.01 & 11.40 & 10.87 & 13.75 \\
\hline W2-F12 & $(\mathrm{T})$ & 1.73 & -2.74 & -0.65 & -0.32 & 0.11 \\
\hline W2-F12 & Inner-shell & 0.60 & 0.84 & 0.51 & 0.86 & 0.76 \\
\hline W2-F12 & Scalar rel. & -0.06 & -0.11 & -0.07 & -0.12 & -0.07 \\
\hline W3.2 & $\mathrm{T}-(\mathrm{T})$ & -0.06 & -0.38 & -0.59 & -0.31 & -0.12 \\
\hline W3.2 & (Q) & 0.48 & -0.33 & -0.22 & 0.11 & 0.36 \\
\hline W2-F12 & $\operatorname{CCSD}(\mathrm{T}) / \mathrm{CBS}^{a}$ & -2.73 & 11.89 & 13.85 & 13.19 & 16.53 \\
\hline \multirow[t]{2}{*}{ W3-F12 } & $\operatorname{CCSDT}(\mathrm{Q}) / \mathrm{CBS}^{b}$ & -2.31 & 11.18 & 13.04 & 12.94 & 16.77 \\
\hline & $\mathrm{ZPVE}^{c}$ & 2.97 & 2.35 & 1.08 & 2.46 & 3.24 \\
\hline W2-F12 & $\operatorname{CCSD}(\mathrm{T}) / \mathrm{CBS}^{d}$ & 0.24 & 14.24 & 14.94 & 15.64 & 19.77 \\
\hline W3-F12 & $\operatorname{CCSDT}(\mathrm{Q}) / \mathrm{CBS}^{e}$ & 0.66 & 13.53 & 14.12 & 15.40 & 20.01 \\
\hline Other $^{f}$ & ae-CCSD $(\mathrm{T}) /$ cc-pVTZ ${ }^{g}$ & 2.01 & 13.82 & $\mathrm{~N} / \mathrm{A}$ & 16.78 & 21.14 \\
\hline Other $^{h}$ & M06-2X/cc-pVTZ & -0.60 & 18.00 & $\mathrm{~N} / \mathrm{A}$ & 20.70 & 17.80 \\
\hline Other $^{i}$ & $\operatorname{CCSD}(\mathrm{T}) / \mathrm{cc}-\mathrm{pVQZ} / / \mathrm{B} 3 \mathrm{LYP} / 6-311 \mathrm{G}(\mathrm{d}, \mathrm{p})^{j}$ & -1.50 & 11.90 & 13.80 & 13.50 & 17.70 \\
\hline
\end{tabular}

\footnotetext{
${ }^{a}$ Relativistic, all-electron CCSD(T)/CBS relative energies at the bottom of the well $\left(\Delta E_{e}\right) .{ }^{b}$ Relativistic, all-electron CCSDT $(\mathrm{Q}) / \mathrm{CBS}$ relative energies at the bottom of the well $\left(\Delta E_{e}\right) .{ }^{c}$ fc-CCSD (T)/cc-pVTZ harmonic ZPVEs scaled by a factor of 0.9868 (see ref. [72] for details). ${ }^{d}$ Relativistic, all-electron $\operatorname{CCSD}(\mathrm{T}) / \mathrm{CBS}$ relative energies at $0 \mathrm{~K}\left(\Delta E_{0}\right) .{ }^{e}$ Relativistic, all-electron $\operatorname{CCSDT}(\mathrm{Q}) / \mathrm{CBS}$ relative energies at $0 \mathrm{~K}\left(\Delta E_{0}\right) .{ }^{f}$ Non-relativistic, all-electron $\operatorname{CCSD}(\mathrm{T}) / \mathrm{cc}-\mathrm{pVTZ}$ relative energies at $0 \mathrm{~K}\left(\Delta E_{0}\right)$ taken from ref. [12]. ${ }^{g}$ Note that the ZPVEs were calculated at the ae-CCSD/DZP level of theory. ${ }^{h}$ Isomerization energies at $0 \mathrm{~K}\left(\Delta E_{0}\right)$ taken from ref. [6]. ${ }^{i}$ Isomerization energies at $0 \mathrm{~K}\left(\Delta E_{0}\right)$ taken from ref. [21]. ${ }^{j}$ Note that the ZPVEs were calculated at the B3LYP/6-311G(d,p) level of theory.
} 
Table 2: Total atomization energies (TAEs; in $\mathrm{kcal} \mathrm{mol}^{-1}$ ) and $\% \mathrm{TAE}[(\mathrm{T})]$ values of $\mathrm{C}_{5} \mathrm{H}_{2}$ isomers at different levels.

\begin{tabular}{|c|c|c|c|c|c|c|c|}
\hline & \multirow[b]{2}{*}{ Isomer } & \multicolumn{3}{|c|}{ cc-pVDZ } & \multicolumn{3}{|c|}{ cc-pVTZ } \\
\hline & & CCSD & $\operatorname{CCSD}(\mathrm{T})$ & $\% \operatorname{TAE}[(\mathrm{T})]^{a}$ & CCSD & $\operatorname{CCSD}(\mathrm{T})$ & $\% \operatorname{TAE}[(\mathrm{T})]^{a}$ \\
\hline \multirow[t]{6}{*}{ TAE } & 1 & 712.00 & 729.70 & 2.42 & 753.68 & 775.39 & 2.80 \\
\hline & 2 & 715.74 & 732.19 & 2.25 & 757.25 & 777.31 & 2.58 \\
\hline & 3 & 700.93 & 721.48 & 2.85 & 740.26 & 764.60 & 3.18 \\
\hline & 4 & 697.04 & 715.51 & 2.58 & 738.77 & 761.01 & 2.92 \\
\hline & 5 & 701.58 & 719.81 & 2.53 & 740.92 & 762.90 & 2.88 \\
\hline & 6 & 696.07 & 714.17 & 2.53 & 736.80 & 758.40 & 2.85 \\
\hline
\end{tabular}


Table 3: Optimal geometry parameters ( $\AA$ and degrees), dipole moment (Debye), rotational constants $(\mathrm{MHz})$, and centrifugal distortion constants $(\mathrm{MHz}$; based on the A-reduced Hamiltonian representation) for the singlet ground electronic state $\left(\widetilde{X}^{1} A_{1}\right)$ of isomer $\mathbf{4}$ calculated at different levels.

\begin{tabular}{|c|c|c|c|c|c|c|}
\hline \multirow[b]{2}{*}{ Parameter } & \multicolumn{3}{|c|}{ cc-pVDZ } & \multicolumn{3}{|c|}{ cc-pVTZ } \\
\hline & $\mathrm{MP} 2$ & CCSD & $\operatorname{CCSD}(\mathrm{T})$ & $\mathrm{MP} 2$ & CCSD & $\operatorname{CCSD}(\mathrm{T})$ \\
\hline $\mathrm{R}\left(\mathrm{C}_{1} \mathrm{C}_{2} ; \mathrm{C}_{2} \mathrm{C}_{3}\right)$ & 1.3899 & 1.3973 & 1.3946 & 1.3686 & 1.3760 & 1.3741 \\
\hline $\mathrm{R}\left(\mathrm{C}_{1} \mathrm{C}_{4} ; \mathrm{C}_{3} \mathrm{C}_{5}\right)$ & 1.2448 & 1.2392 & 1.2496 & 1.2286 & 1.2209 & 1.2305 \\
\hline $\mathrm{R}\left(\mathrm{C}_{4} \mathrm{H}_{6} ; \mathrm{C}_{5} \mathrm{H}_{7}\right)$ & 1.0769 & 1.0781 & 1.0802 & 1.0623 & 1.0621 & 1.0641 \\
\hline$\theta\left(\mathrm{C}_{1} \mathrm{C}_{2} \mathrm{C}_{3}\right)$ & 119.56 & 118.59 & 119.84 & 121.53 & 120.32 & 121.49 \\
\hline$\theta\left(\mathrm{C}_{2} \mathrm{C}_{1} \mathrm{C}_{4} ; \mathrm{C}_{2} \mathrm{C}_{3} \mathrm{C}_{5}\right)$ & 170.69 & 171.58 & 168.89 & 172.21 & 173.04 & 171.69 \\
\hline$\theta\left(\mathrm{C}_{1} \mathrm{C}_{4} \mathrm{H}_{6} ; \mathrm{C}_{3} \mathrm{C}_{5} \mathrm{H}_{7}\right)$ & 175.71 & 175.90 & 170.15 & 177.42 & 177.83 & 175.72 \\
\hline Dipole moment & 2.1947 & 2.2066 & 2.2393 & 2.1077 & 2.1253 & 2.0825 \\
\hline$A_{e}$ & 42186.62 & 39235.96 & 44382.21 & 44183.77 & 40935.11 & 44468.31 \\
\hline$B_{e}$ & 2643.18 & 2672.70 & 2601.15 & 2695.85 & 2733.38 & 2675.36 \\
\hline$C_{e}$ & 2487.34 & 2502.25 & 2457.14 & 2540.82 & 2562.29 & 2523.54 \\
\hline$\Delta_{J}$ & $0.1123 \times 10^{-2}$ & $0.1168 \times 10^{-2}$ & $0.1137 \times 10^{-2}$ & $0.1206 \times 10^{-2}$ & $0.1220 \times 10^{-2}$ & $0.1193 \times 10^{-2}$ \\
\hline$\Delta_{K}$ & 12.1330 & 9.1424 & 16.5609 & 14.0108 & 9.9841 & 14.7530 \\
\hline$\Delta_{J K}$ & -0.2049 & -0.1810 & -0.2416 & -0.2294 & -0.1937 & -0.2339 \\
\hline$\delta_{J}$ & $0.2522 \times 10^{-3}$ & $0.2672 \times 10^{-3}$ & $0.2560 \times 10^{-3}$ & $0.2673 \times 10^{-3}$ & $0.2750 \times 10^{-3}$ & $0.2642 \times 10^{-3}$ \\
\hline$\delta_{K}$ & $0.6558 \times 10^{-2}$ & $0.6338 \times 10^{-2}$ & $0.7174 \times 10^{-2}$ & $0.7177 \times 10^{-2}$ & $0.6713 \times 10^{-2}$ & $0.7193 \times 10^{-2}$ \\
\hline
\end{tabular}


Table 4: Harmonic vibrational frequencies $\left(\mathrm{cm}^{-1}\right)$, IR intensities $\left(\mathrm{km} \mathrm{mol}^{-1}\right)$ and isotopic shifts $\left({ }^{12} \mathrm{C}-{ }^{13} \mathrm{C}\right)$ in harmonic vibrational frequencies $\left(\mathrm{cm}^{-1}\right)$ for the ground electronic state $\left(\widetilde{X}^{1} A_{1}\right)$ of isomer 4 calculated at the fc-CCSD $(\mathrm{T}) / \mathrm{cc}-\mathrm{pVTZ}$ level of theory.

\begin{tabular}{|c|c|c|c|c|c|c|}
\hline \multirow[t]{2}{*}{ Mode } & \multicolumn{3}{|c|}{ Isomer 4} & \multicolumn{3}{|c|}{${ }^{12} \mathrm{C}-{ }^{13} \mathrm{C}$} \\
\hline & symmetry & frequency & intensity & $\mathrm{C}(1)$ & $\mathrm{C}(2)$ & $\mathrm{C}(4)$ \\
\hline 1 & $a_{1}$ & 128 & 2 & 0 & 1 & 2 \\
\hline 2 & $b_{2}$ & 291 & 28 & 2 & 0 & 2 \\
\hline 3 & $b_{1}$ & 297 & 1 & 5 & 3 & 1 \\
\hline 4 & $a_{2}$ & 305 & - & 3 & - & 1 \\
\hline 5 & $a_{1}$ & 329 & 108 & 0 & 0 & 1 \\
\hline 6 & $b_{2}$ & 436 & 3 & 5 & 0 & 0 \\
\hline 7 & $a_{1}$ & 561 & 4 & 7 & 3 & 2 \\
\hline 8 & $a_{2}$ & 768 & - & 1 & - & 6 \\
\hline 9 & $b_{1}$ & 769 & 35 & 0 & 0 & 0 \\
\hline 10 & $a_{1}$ & 832 & 3 & 3 & 15 & 6 \\
\hline 11 & $b_{2}$ & 1274 & 88 & 1 & 36 & 6 \\
\hline 12 & $b_{2}$ & 1974 & 186 & 33 & 3 & 14 \\
\hline 13 & $a_{1}$ & 2025 & 1 & 15 & 0 & 10 \\
\hline 14 & $b_{2}$ & 3451 & 110 & 0 & 0 & 15 \\
\hline 15 & $a_{1}$ & 3454 & 26 & 0 & 0 & 1 \\
\hline
\end{tabular}

